\title{
Laparoscopic and natural orifice transluminal restorative proctocolectomy: no abdominal incision for specimen extraction or ileostomy
}

\author{
Cuneyt Kayaalp, Mehmet Ali Yagci, Vural Soyer \\ Department of Surgery, Inonu University, Malatya, Turkey
}

Videosurgery Miniinv 2016; 11 (2): 115-120 DOI: $10.5114 /$ wiitm.2016.59578

\begin{abstract}
The aim of this study was to demonstrate the feasibility of laparoscopic restorative proctocolectomy (LRPC) without additional abdominal incisions. Two sisters with familial adenomatous polyposis were enrolled. The colon and rectum were mobilized entirely through the five abdominal trocars. The terminal ileum and distal rectum were transected with endoscopic staplers. The entire colorectal specimen was extracted transanally. A circular stapler anvil was introduced transanally. The J-pouch was created intracorporeally. The rectal stump was re-closed and a pouch-anal anastomosis was created using a circular stapler. We used a transanal tube for decompression of the pouch instead of a diverting ileostomy. The patients were discharged on the $10^{\text {th }}$ and $12^{\text {th }}$ days uneventfully. Both were doing well with their pouches after 18.5 and 12.1 months of follow-up. With the help of transanal specimen extraction and transanal tube decompression, additional abdominal incisions can be avoided following LRPC.
\end{abstract}

Key words: laparoscopic restorative proctocolectomy, natural orifice surgery, natural orifice transendoluminal surgery, natural orifice specimen extraction, ileostomy.

\section{Introduction}

Laparoscopic-assisted, hand-assisted and total laparoscopic restorative proctocolectomy (LRPC) have all been described in the management of familial adenomatous polyposis (FAP) and ulcerative colitis $[1,2]$. Despite the satisfactory results following these procedures, an abdominal wall incision (a mini-laparotomy) was always required to retrieve the specimen or for the other interventions. In addition, an ileostomy was usually necessary.

Natural orifice specimen extraction (NOSE) eliminates the need for abdominal wall incision for specimen extraction and minimizes the risk of wound complications. It provides fewer incisional hernias, faster recovery and less postoperative pain [3-5].
On the other hand, avoiding an ileostomy in selected cases by using a transanal decompression tube during LRPC is a known procedure [6].

Here, we combined both advanced techniques, and aimed to demonstrate the feasibility of the LRPC without any abdominal incisions. To the best of our knowledge, these were the first human cases of LRPC with transanal specimen extraction without ileostomy. Thus, the procedure was registered at www.researchregistery.com (\#301).

\section{Case reports}

Two sisters, aged 41 and 32 years, suffered from FAP and were advised to undergo LRPC. Their medical histories and physical examinations were not

\section{Address for correspondence}

Prof. Cuneyt Kayaalp, Department of Surgery, Inonu University, 44315 Malatya, Turkey, phone: +90 4223774001

e-mail: cuneytkayaalp@hotmail.com 


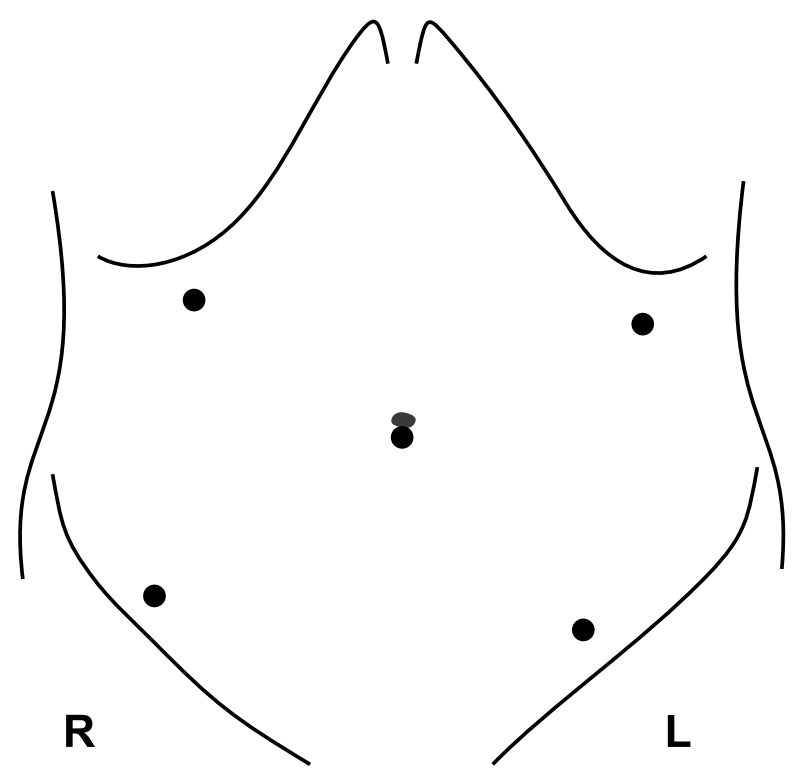

Figure 1. Trocar sites

significant. They had two brothers, and both had a history of open restorative proctocolectomies for FAP. The American Society of Anesthesiologists (ASA) scores of the sisters were I and their biochemical parameters were within normal ranges. The details of this new technique were explained to both women and the appropriate consent was obtained. Prophylactic intravenous antibiotics were given one hour prior to the operation, and antithrombotic prophylaxis was administered via low-molecular-weight heparin and elastic stockings. Both patients underwent bowel preparation the day before surgery. A nasogastric tube, a urinary catheter, and a central venous catheter were inserted before the procedure.

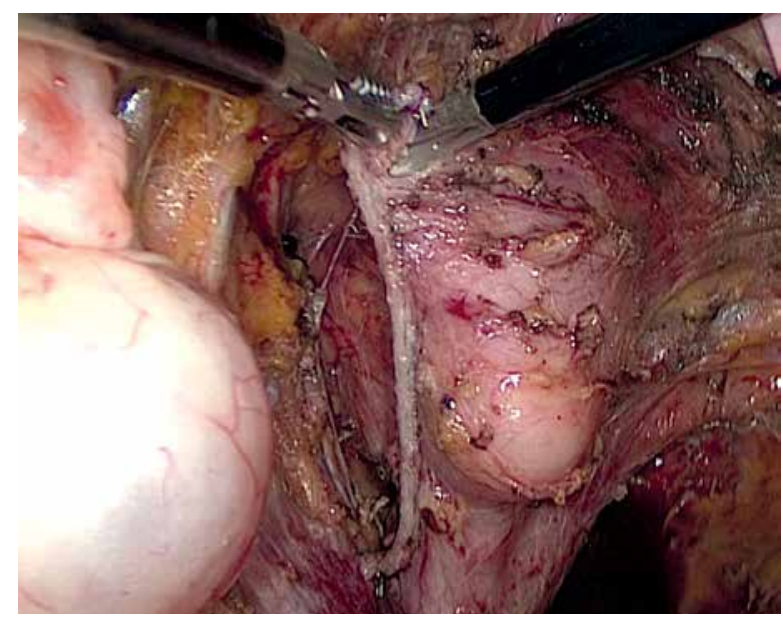

Photo 1. Opening the rectal stump

\section{Procedure}

Two monitors were used on the right and left sides of the patients. The patients were placed in a semilithotomy position. Pneumoperitoneum was created using a Veress needle through the umbilicus, and five trocars were positioned (Figure 1). The procedure was initiated via the mobilization of the rectosigmoid towards the descending colon. The inferior mesenteric vessels were ligated, and were divided using a $10 \mathrm{~mm}$ LigaSure vessel-sealing system (ForceTriad, Covidien, Boulder, CO, USA). The middle colic vessels were divided, and the splenic flexure and transverse colon were mobilized. The ascending colon and hepatic flexure were mobilized, and the distal ileum was transected using an endoscopic $60 \mathrm{~mm}$ linear stapler (Endo GIA, Covidien, Mansfield, MA, USA). A medial to lateral approach was used for the mesenteric dissections. Lastly, the rectum was transected using an endoscopic $60 \mathrm{~mm}$ linear stapler $2-3 \mathrm{~cm}$ proximal to the anal canal. The specimen was completely free in the abdomen. Following transanal lavage with povidone-iodine and normal saline, the rectal stump was opened using Endo shears and electrocautery (Photo 1). The anal canal was dilated up to two fingers (Photo 2), and the specimen was extracted transanally using an ovary clamp (Photos 3 and 4). Then a $15 \mathrm{~cm}$ length J-pouch to the terminal ileum was fashioned intracorporeally (Photo 5). The anvil of a $31 \mathrm{~mm}$ circular stapler (Autosuture, Tyco Healthcare Group LP, Norwalk, Connecticut) was introduced into the abdomen transanally (Photo 6), inserted into the pouch and secured with intracorporeal purse-string sutures (Photo 7). The opened rectal stump was closed us-

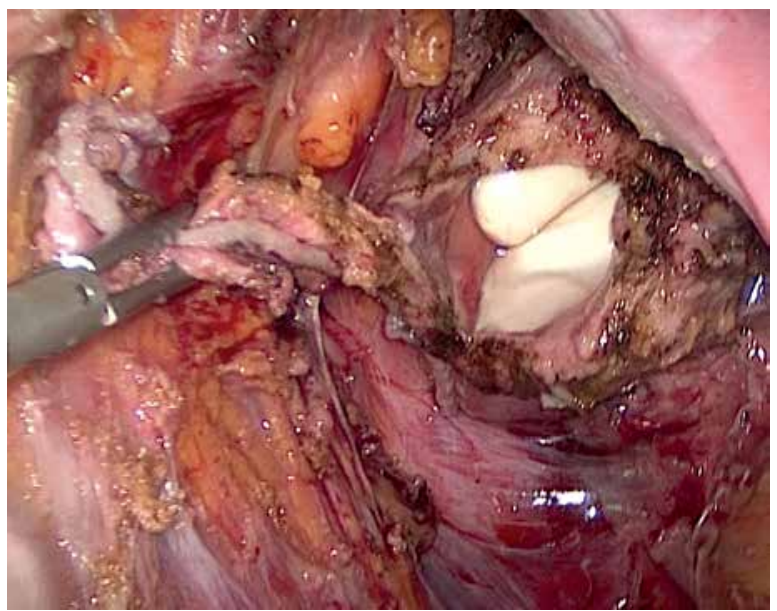

Photo 2. Dilatation of the anal canal 


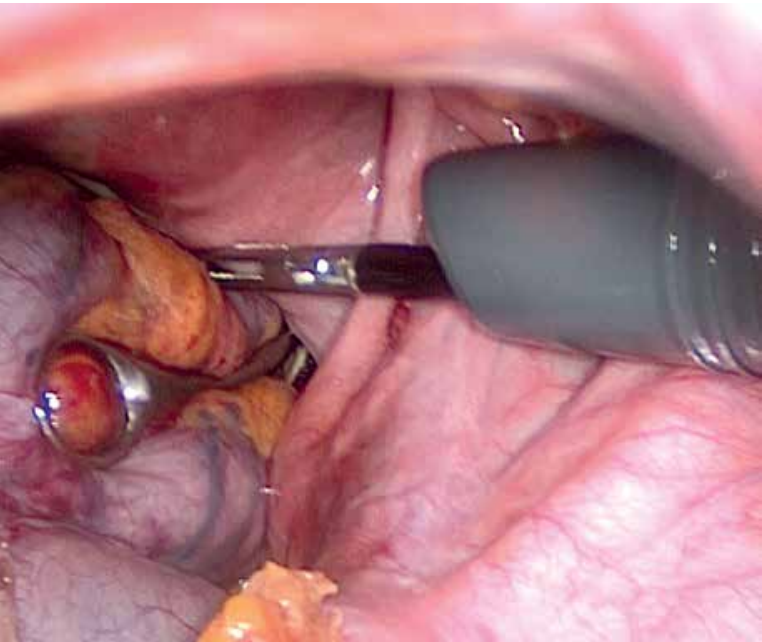

Photo 3. Removing the total proctocolectomy material using an ovary clamp

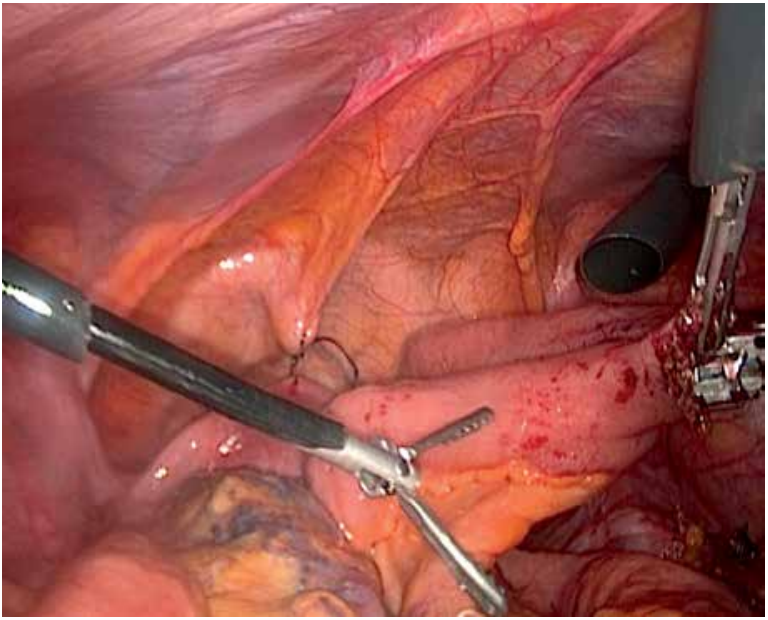

Photo 5. Creation of the J-pouch intracorporeally

ing a laparoscopic stapler (Photo 8). Finally, the head of the circular stapler was passed through the anus (Photo 9) and a circular anastomosis was created (Photo 10). We did not perform a diverting ileostomy, but preferred a transanal tube $(28 \mathrm{~mm}$ Pezzer tube) to decompress the pouch.

\section{Results}

The operations took 420 and 510 min with approximately 100 and $300 \mathrm{ml}$ of blood loss. Neither additional port placement nor extension of the trocar site was required. All the procedures were completed entirely through the five $(5-12 \mathrm{~mm})$ abdominal trocars. The pouch-anal anastomoses were created $4 \mathrm{~cm}$ away from the anal verge. There were no intra-

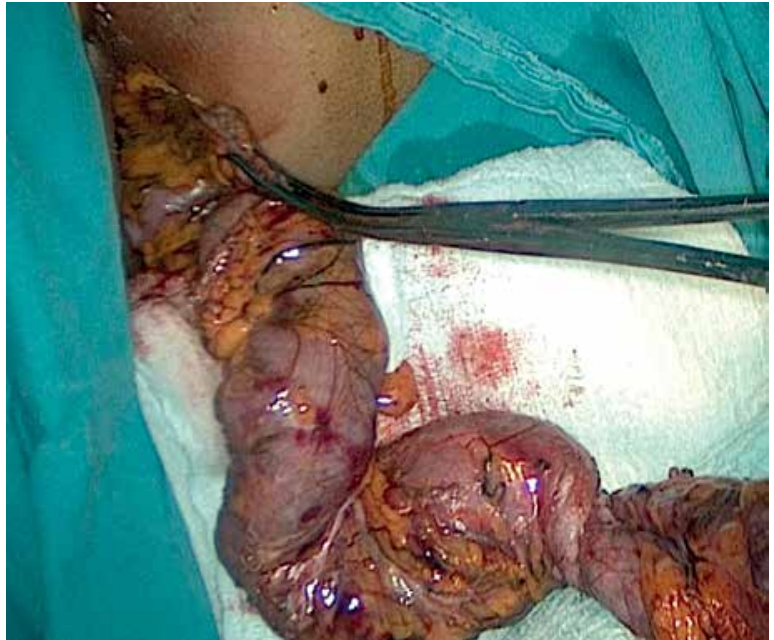

Photo 4. Extraction of the specimen through the anus

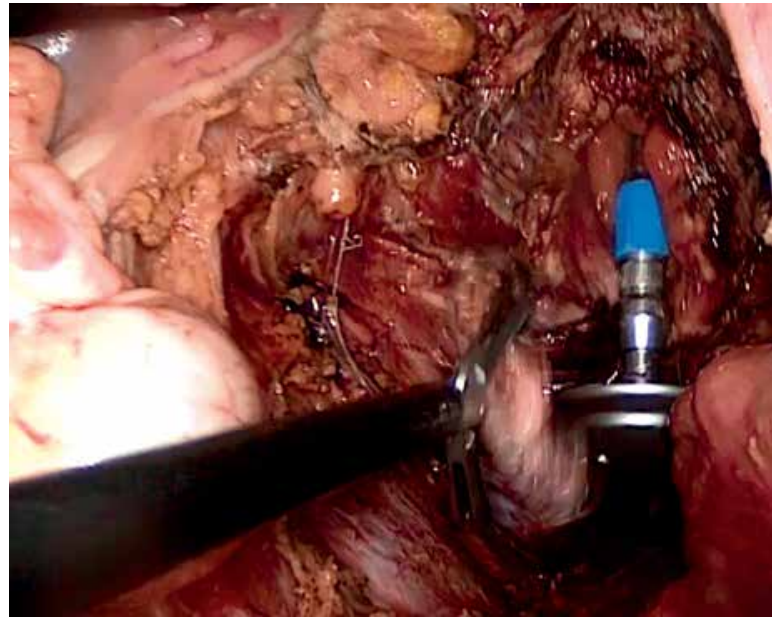

Photo 6. Pushed in stapler anvil through the anus

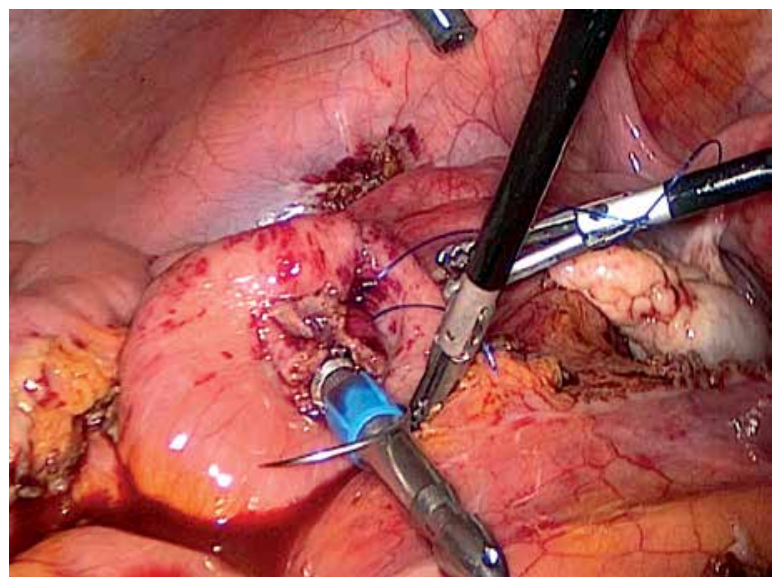

Photo 7. Intracorporeal purse-string suture around the anvil in the pouch 


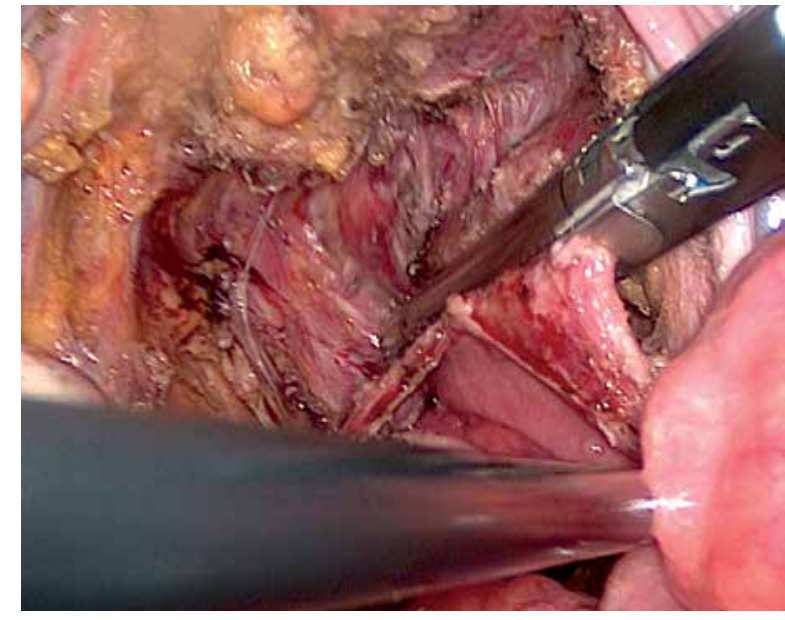

Photo 8. Re-closing the distal stump

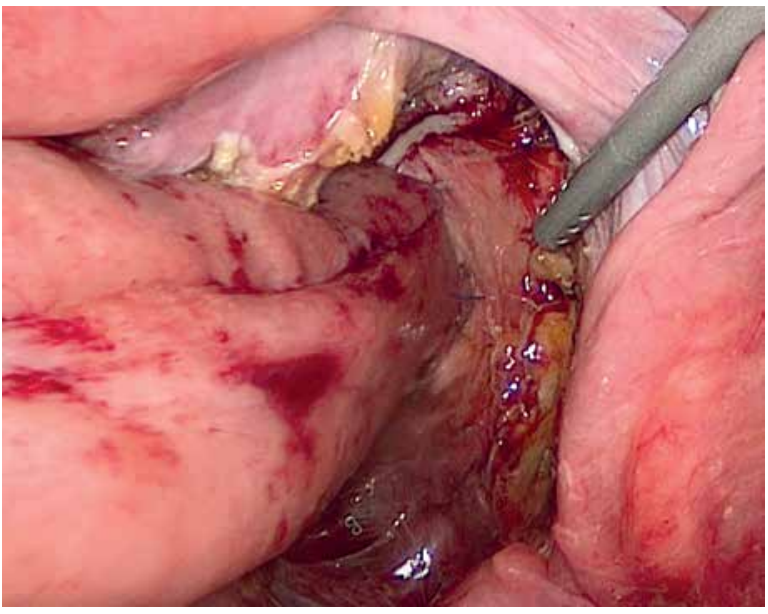

Photo 10. Pouch anal anastomosis

operative or early postoperative complications, and the passage of flatus and feces from the transanal tube of each patient occurred on the third day after the operation. The transanal tubes were irrigated using $10 \mathrm{ml}$ saline four times a day to prevent clogging. Total parenteral nutrition was performed for 7 days. A liquid diet was introduced on the seventh postoperative day and was increased gradually. After the enteral diet was tolerated, the transanal tubes were removed on the ninth and eighth postoperative days. Postoperative courses were uneventful, and the patients were discharged on the $10^{\text {th }}$ and $12^{\text {th }}$ postoperative days. Histopathologic examinations revealed FAP without malignancy. During the follow-up, the first patient suffered from an intra-abdominal abscess after 3 months and required abscess drainage. Both patients were doing well with

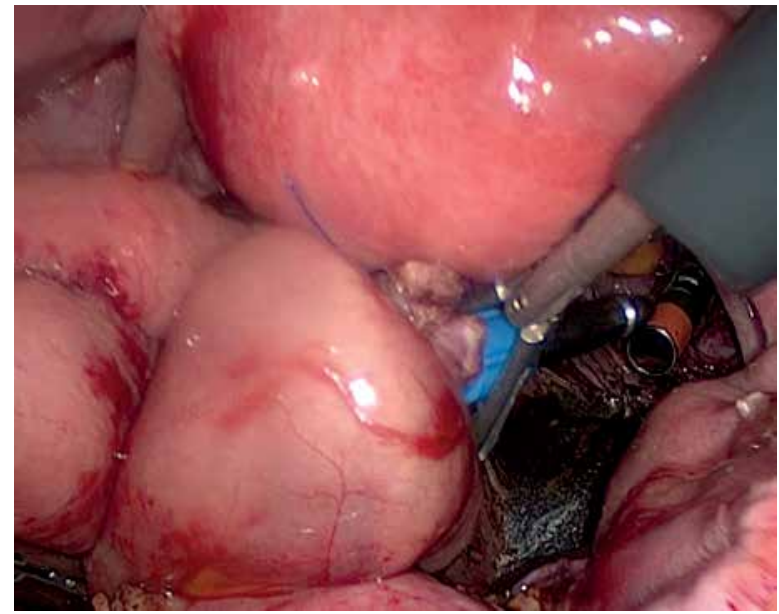

Photo 9. Alignment of the shaft and the anvil of the circular stapler

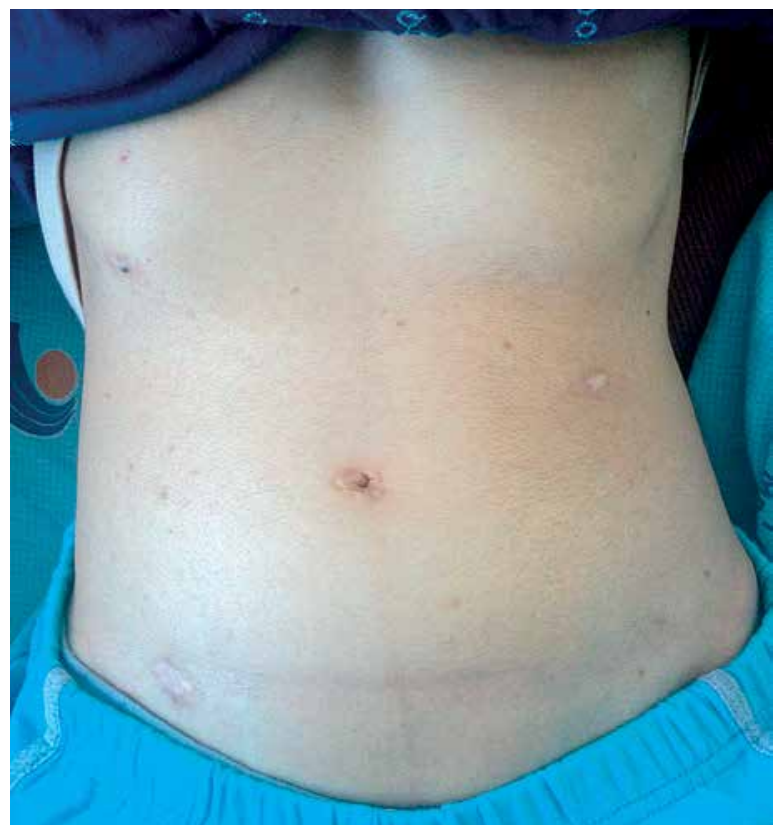

Photo 11. Abdominal view of the patient 9 months after the operation

their pouches after 18.5 and 12.1 month follow-ups (Photo 11). They had good anal continence without soiling, even at night. On their last visit, the patients had a mean of 6 and 7 defecations per day, respectively.

\section{Discussion}

Transanal specimen extraction has been previously described for left or right-sided colon resec- 
tions [7, 8], total colectomies [9] and rectal resections [10]. To the best of our knowledge, transanal specimen extraction for a total proctocolectomy has not been described previously. In general, the potential advantages of natural orifice surgery are lower risks of incision-related complications such as wound infections, postoperative pain and incisional hernias, as well as better cosmesis. Omitting the ileostomy increases the advantages of natural orifice surgery. Choosing the natural orifice extraction site for colorectal specimens is a matter of debate, and there are two options: the vagina and the anus. Transvaginal extraction of the proctocolectomy specimen has been described by Palanivelu et al. [11], with the conclusion that the transanal route can cause damage to the internal anal sphincter in cases of handsewn anastomosis. We agree that there can be overstretching on the anal sphincter during a hand-sewn anastomosis. However, we preferred the double stapling technique to the hand-sewn anastomosis. It has been shown to be a safe and effective method for pouch-anal anastomosis with good anal sphincter functions [12]. Transvaginal extraction is limited to female patients, and the transanal extraction of a colorectal specimen avoids any additional trauma to any innocent organ $[9,13]$. We believe that the transvaginal route can be a good alternative for right-sided colonic resections [14, 15]. Lastly, transvaginal extraction of the specimen is not always suitable for patients of childbearing age, teenagers or virgins. Our second patient was a virgin, and she refused transvaginal access. Cultural sensitivity regarding the use of the vagina for surgical specimen extraction in virgins can be a problem, particularly in Third World countries, but also in the most promiscuous cultures.

Restorative proctocolectomy was first described using a diverting ileostomy due to the risk of anastomotic leakage and pelvic sepsis. Over the course of time, surgeons learnt that routine use of a diverting ileostomy was not necessary. A diverting stoma was justified when there were certain risk factors, such as steroid use and hand-sewn pouch anal anastomosis, but there have been no universally accepted criteria. Recently, a five-point predictive nomogram for the omission of an ileostomy during pouch surgery was suggested by the Cleveland Clinic [6]. They found five significant criteria to be associated with ileostomy omission: stapled anastomosis, no preoperative corticosteroid use, familial adenomatous pol- yposis diagnosis, female gender, and age at surgery less than 26 years. With high scores, the omission of proximal diversion had no significant effect on postoperative adverse events. Both of our patients had high points (45-50; the maximum score for the nomogram is 54) for the nomogram, and had a good chance of stoma-free surgery. We relied on the nomogram and omitted the ileostomies in both cases; however, we placed a transient transanal tube to decompress the pouch lumen in both cases. Transanal tube decompression was not a proven method, and thus we could not suggest it as an alternative to a diverting ileostomy, but we believed that it provided low pressure in the pouch in the early postoperative period. Total parenteral nutrition and nil per os for 1 week prolonged the length of hospital stay as expected, and the patients were discharged on days 10 and 12.

\section{Conclusions}

Laparoscopic restorative proctocolectomy without ileostomy and transanal specimen extraction is a promising technique for some selected patients. It has all the advantages of minimally invasive surgery; moreover, it avoids any kind of mini-laparotomy, including the stoma. Transanal extraction of the colorectal specimen provides natural orifice surgery for both genders.

\section{Conflict of interest}

The authors declare no conflict of interest.

\section{References}

1. Sylla P, Chessin DB, Gorfine SR, et al. Evaluation of one-stage laparoscopic-assisted restorative proctocolectomy at a specialty center: comparison with the open approach. Dis Colon Rectum 2009; 52: 394-9.

2. Polle SW, Dunker MS, Slors JF, et al. Body image, cosmesis, quality of life, and functional outcome of hand-assisted laparoscopic versus open restorative proctocolectomy: long-term results of a randomized trial. Surg Endosc 2007; 21: 1301-7.

3. Solomon D, Shariff AH, Silasi DA, et al. Transvaginal cholecystectomy versus single-incision laparoscopic cholecystectomy versus four-port laparoscopic cholecystectomy: a prospective cohort study. Surg Endosc 2012; 26: 2823-7.

4. Leung AL, Cheung HY, Fok BK, et al. Prospective randomized trial of hybrid NOTES colectomy versus conventional laparoscopic colectomy for left-sided colonic tumors. World J Surg 2013; 37 : 2678-82.

5. Bulian DR, Knuth J, Cerasani N, et al. Transvaginal/transumbilical hybrid-NOTES-versus 3-trocar needlescopic cholecystecto- 
my: short-term results of a randomized clinical trial. Ann Surg 2015; 261: 451-8

6. Lovegrove RE, Tilney HS, Remzi FH, et al. To divert or not to divert: a retrospective analysis of variables that influence ileostomy omission in ileal pouch surgery. Arch Surg 2011; 146: 82-8.

7. Costantino FA, Diana M, Wall J, et al. Prospective evaluation of peritoneal fluid contamination following transabdominal vs. transanal specimen extraction in laparoscopic left-sided colorectal resections. Surg Endosc 2012; 26: 1495-500.

8. Eshuis EJ, Voermans RP, Stokkers PC, et al. Laparoscopic resection with transcolonic specimen extraction for ileocaecal Crohn's disease. Br J Surg 2010; 97: 569-74.

9. Awad ZT. Laparoscopic subtotal colectomy with transrectal ex traction of the colon and ileorectal anastomosis. Surg Endosc 2012; 26: 869-71.

10. Franklin ME Jr, Liang S, Russek K. Integration of transanal specimen extraction into laparoscopic anterior resection with total mesorectal excision for rectal cancer: a consecutive series of 179 patients. Surg Endosc 2013; 27: 127-32.

11. Palanivelu C, Rangarajan M, Jategaonkar PA, Anand NV. An innovative technique for colorectal specimen retrieval: a new era of "natural orifice specimen extraction" (N.O.S.E). Dis Colon Rectum 2008; 51: 1120-4.

12. Kayaalp C, Nessar G, Akoglu M, Atalay F. Elimination of mucosectomy during restorative proctocolectomy in patients with ulcerative colitis may provide better results in low-volume centers. Am J Surg 2003; 185: 268-72.

13. Franklin ME Jr, Liang S, Russek K. Natural orifice specimen extraction in laparoscopic colorectal surgery: transanal and transvaginal approaches. Tech Coloproctol 2013; 17 Suppl 1: S63-7.

14. Kayaalp C, Yagci MA. Laparoscopic right colon resection with transvaginal extraction: a systematic review of 90 cases. Surg Laparosc Endosc Percutan Tech 2015; 25: 284-91.

15. Yagci MA, Kayaalp C, Kutluturk K. Laparoscopic right colectomy with transvaginal extraction in a patient with prior pancreaticoduodenectomy. G Chir 2014; 35: 209-12.

Received: 23.07.2015, accepted: 4.04.2016. 